\title{
THE CANCELLATION PROBLEM FOR FUNCTION FIELDS
}

\author{
JAMES K. DEVENEY
}

(Communicated by William C. Waterhouse)

\begin{abstract}
Let $L$ be a finitely generated extension of $K$. We call $L$ rigid over $K$ if the set of $K$-endomorphisms of $L$ is finite. If $L$ is rigid over $K$ and $x$ is transcendental over $L$, then $L$ is invariant under automorphisms of $L(x)$ over $K$ (Theorem 2). This result is used to show that the cancellation property holds for function fields of varieties of general type in characteristic 0 .
\end{abstract}

Let $L_{1}$ and $L_{2}$ be finitely generated extensions of a field $K$ and let $x_{i}$ be transcendental over $L_{i}, i=1,2$. The Zariski problem [4] or what is more generally known as the cancellation problem asks: if $L_{1}\left(x_{1}\right)=L_{2}\left(x_{2}\right)$, must $L_{1}$ and $L_{2}$ be $K$-isomorphic? In general the answer is no. In [1], the authors construct a family of examples where $L / K$ is of transcendence degree 2 and not rational, yet $L\left(x_{1}, x_{2}, x_{3}\right)$ is $K$-isomorphic to $K\left(x_{4}, x_{5}, x_{6}, x_{7}, x_{8}\right)$. The question remains whether this type of behavior is typical. An application of the main result of this paper shows it to be the exception. Specifically, it is shown that if $L_{1} / K$ is the function field of a variety of general type of characteristic 0 , then the cancellation property is true for $L_{1} / K$.

DEFINITION 1. $L / K$ is called rigid if the set of $K$-endomorphisms of $L$ is finite.

$L$ is rigid over $K$ if and only if the group of automorphisms of $L$ over $K$ is finite and $L$ has no proper endomorphisms over $K$. This follows since once $L / K$ has a proper endomorphism, repeated application will yield an infinite family of proper endomorphisms. For function fields of transcendence degree one over $K, L$ is rigid over $K$ if and only if the genus of $L$ over $K$ is at least two.

THEOREM 2. Let $L$ be a finitely generated extension of $K$ with $x$ transcendental over $L$ and let Aut $_{K} L(x)$ be the group of automorphisms of $L(x)$ which leave $K$ fixed. If $L / K$ is rigid and $\sigma \in \operatorname{Aut}_{K}(L(x))$, then $\sigma(L)=L$.

PROOF. If $L$ is algebraic over $K$, then $L$ is the algebraic closure of $K$ in $L(x)$ and hence $\sigma(L) \subseteq L$. Since $L / K$ is rigid, $\sigma(L)=L$.

Thus we may assume $L$ is not algebraic over $K$, and hence $L$ is infinite. If $\sigma(L) \subseteq(L)$ then as above $\sigma(L)=L$. So assume we have a $\sigma \in \operatorname{Aut}_{K} L(x)$ such that $\sigma(L) \nsubseteq(L)$ and we obtain a contradiction. Using $\sigma$ we obtain a diagram

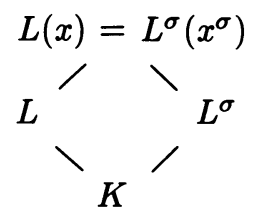

Received by the editors December 5, 1986 and, in revised form, April 7, 1987. 1980 Mathematics Subject Classification (1985 Revision). Primary 12F20. 
Let $a \in L$ and consider the $(x-a)$-place of $L(x)$ whose residue field is $L$. Let $L_{a}^{\sigma}$ be the residue field of $L^{\sigma}$ under the restriction of the place and note we have $L \supseteq L_{a}^{\sigma} \supseteq K$. According to the ruled residue theorem [5], either $L$ is algebraic over $L_{a}^{\sigma}$ or $L$ is ruled over the algebraic closure of $L_{a}^{\sigma}$ in $L$. But if $L=L_{1}(z)$ with $z$ transcendental over $L_{1}$, then $L$ is $K$-isomorphic to $L_{1}\left(z^{2}\right)$, which is not possible since $L / K$ is rigid. Thus $L$ must be algebraic over $L_{a}^{\sigma}$. Since the transcendence degree of $L^{\sigma} / K$ has not dropped under the place, the place must give an isomorphism of $L^{\sigma}$ into $L$. Once again since $L / K$ is rigid, this isomorphism must be onto $L$. Thus each element $a \in L$ determines an isomorphism (over $K$ ), call it $\gamma_{a}$, of $L^{\sigma}$ onto $L$. Thus each $\gamma_{a} \circ \sigma$ is an automorphism of $L / K$. Since $L$ is rigid over $K$ and infinite, some infinite subset of these, say $\gamma_{a_{i}} \circ \sigma$ where $i \in N$, all determine the same automorphism of $L$. Thus the $\gamma_{a_{i}}$ must all define the same isomorphism of $L^{\sigma}$ onto $L$. Let $\alpha \in L^{\sigma} \backslash L$. Then $\alpha=r(x)$ is a nonconstant rational function with coefficients in $L$. Thus $r(x)-r\left(a_{1}\right)$ is also a nonconstant rational function. Since $\gamma_{a_{i}}(r(x))=\gamma_{a_{1}}(r(x)), r(x)-r\left(a_{1}\right)$ has an infinite number of distinct roots, a contradiction. Thus $L$ is invariant under $\operatorname{Aut}_{K} L(x)$.

COROLLARY 3 . Let $L_{i}$ be finitely generated over $K$ and $x_{i}$ transcendental over $L_{i}, i=1,2$, and suppose $L_{1}\left(x_{1}\right)=L_{2}\left(x_{2}\right)$. If $L_{1} / K$ is rigid, then $L_{2}=L_{1}$.

PROOF. Assume $L_{2} \neq L_{1}$. Then $L_{1} L_{2}$ properly contains both $L_{1}$ and $L_{2}$ and hence by Luroth's theorem $L_{1} L_{2}$ is simple transcendental over both $L_{1}$ and $L_{2}$. Thus we may assume $L_{1} L_{2}=L_{2}\left(x_{2}\right)$. Thus automorphisms of $L_{2}\left(x_{2}\right) / L_{2}$ are determined by where they map the elements of $L_{1}$. Since $L_{1} / K$ is rigid, $L_{1}$ is invariant under these automorphisms (Theorem 3). Thus the group of automorphisms of $L_{2}\left(x_{2}\right)$ over $L_{2}$ is finite. Thus $L_{2}$ is finite and both $L_{1}$ and $L_{2}$ are the algebraic closure of $K$ in $L_{2}\left(x_{2}\right)$.

COROLLARY 4. Let $L_{i}$ be finitely generated over $K$ and $x_{i}$ transcendental over $L_{i}, i=1,2$, and suppose $L_{1}\left(x_{1}\right)=L_{2}\left(x_{2}\right)$. If $L_{1} / K$ is the function field of $a$ variety of general type of characteristic 0 , then $L_{1}=L_{2}$.

PROOF. Let $Y$ be a smooth projective variety over $K$ whose function field is $L_{1}$. Since the characteristic of $K$ is 0 such a model exists. There is 1-1 correspondence between $K$-endomorphisms of $L_{1}$ and separable dominant rational maps from $Y$ onto $Y$. [3, Theorem 2, p. 286] shows that the set of such maps is finite and hence $L_{1} / K$ is rigid. Corollary 3 now applies.

\section{REFERENCES}

1. A. Beauville, J.-L. Colliot-Thélène, J.-J. Sansuc, H. P. F. Swinnerton-Dyer, Variétés stablement rationnelles non rationnnelles, Ann. of Math. (2) 121 (1985), 283-318.

2. J. Deveney, Automorphism groups of ruled function fields and a problem of Zariski, Proc. Amer. Math. Soc. 90 (1984), 178-180.

3. M. Martin-Deschamps and R. Lewin-Menegaux, Applications rationnelles separables dominantes sur une variete de type general, Bull. Soc. Math. France 106 (1978), 279-287.

4. M. Nagata, $A$ theorem on valuation rings and its applications, Nagoya Math. J. 29 (1967), 85-91.

5. J. Ohm, The ruled residue theorem for simple transcendental extensions of valued fields, Proc. Amer. Math. Soc. 89 (1983), $16-18$.

Department of Mathematical Sciences, Virginia Commonwealth UniverSITY, RICHMOND, VIRGINIA 23284 